\title{
An unknown Transit technique for Solving Generalized Trapezoidal Intuitionistic Fuzzy Transportation Problems using Centroid of Centroids
}

\author{
Indira Singuluri ${ }^{a}$, N. Ravi Shankar ${ }^{\text {b }}$ \\ a'Vignan's Institute of Information Technology (A), Visakhapatnam, Andhra Pradesh \\ ${ }^{\mathrm{b}}$ GITAM (Deemed to be University), Visakhapatnam, Andhra Pradesh. \\ andira.singuluri@gmail.com, bdrravi68@gmail.com
}

Article History: Received: 10 November 2020; Revised 12 January 2021 Accepted: 27 January 2021; Published online: 5 April 2021

\begin{abstract}
In the present day by day life circumstances TP we habitually face the circumstance of unreliability in addition to unwillingness due to various unmanageable segments. To deal with unreliability and unwillingness multiple researchers have recommended the intuitionistic fuzzy (IF) delineation for material. This paper proposes the approach used by generalized trapezoidal intuitionistic fuzzy number to solve these transport problem, i.e. capacity and demand are considered as real numbers and charge of transport from origin to destination is considered as generalized trapezoidal intuitionistic fuzzy numbers as charge of product per unit. The generalized trapezoidal intuitionistic fuzzy numbers ranking function is used on the basis of IFN'S centroid of centroids. Through the traditional optimization process, we generate primary basic feasible solution and foremost solution. The numerical illustration shows efficacy of technique being suggested. A fresh technique is implemented to seek foremost solution using ranking function of a fuzzy TP of generalized trapezoidal intuitionistic fuzzy number. Without finding a IBFS, this approach explicitly provides optimal solution for GTrIFTP. Finally, for ranking function we apply a proposed GTrIFTP method illustrated Numerical example.
\end{abstract}

Keywords: IFN, TrIFN, Intuitionistic Optimum solution, Trapezoidal intuitionistic fuzzy transportation problem.

\section{Introduction}

Fuzzy set (FS) theory was first invented by Zadeh [11] has been involved effective in different fields. The concept fuzzy mathematical programming was invented by Tanaka et al in 1947 the framework of fuzzy decision of Bellman et al [2].The concept of Intuitionistic fuzzy sets (IFS's) suggested by Atanassov [1] is found to be hugely useful to deal with ambiguity. The IFS's separate proportion integration (fulfillment level) and proportion non-participation (non-fulfillment level) of an element in the set. IFS's assist constrained to agree proportion fulfillment, proportion of non-fulfillment and proportion of uncertainty for consignment and assist to mould decision about intensity of approval and non-approval for TC in any TP. Owing upon execution of IFS theory enhance fit attractive in regulating obstacles. Consequently keen exceed to avail IFS in contrast with FS to review unfaithfulness. In Ismail Mohideen et al [4], look over a relative swot on TP in fuzzy domain. Stephen et al. [6] investigated a method to solve fuzzy transportation problem (FTP) by taking trapezoidal fuzzy numbers. So, many authors used IFS's in different regenerate obstacles. Chakraborty et al. [3] introduced computational operations of IFS's. Multiple researchers further devised with IFS's. Intuitionistic trapezoidal fuzzy numbers are introduced in Wang et al. [10], which are extending of intuitionistic triangular fuzzy numbers. Intuitionistic triangular fuzzy numbers and intuitionistic trapezoidal fuzzy numbers are extending of intuitionistic fuzzy sets in another way, which extends discrete set to continuous set, and they are extending of fuzzy numbers. Intuitionistic trapezoidal fuzzy weighted arithmetic averaging operators and weighted geometric averaging operators are introduced by Wang et al. [8][9]. PardhaSaradhi et al. [5] defined ordering of IFN's using centroid of centroids of IFN. We extended above paper [5] by assuming transportation problem. However assumed TP is solved by using proposed method.

Rest of article is organized as follows: Section 2 Preliminaries deals with some basic definitions, section 3 provides Ranking function of GTrIFN, section 4 deals with mathematical formulation and proposed method, section 5 consists Numerical example, finally conclusion is given in section 6 .

\section{Preliminaries}

In this segment a few preliminaries and computations are discussed. 


\section{Intuitionistic Fuzzy Set (IFS):}

An IFS $A$ in $X$ is detailed as object of following form

$A^{I F S}=\left\{\left(x, \mu_{A^{I F S}}^{-}(x), v_{A^{I F S}}(x)\right): x \in X\right\}$

where the functions $\mu_{A^{I F S}}^{-}: X \rightarrow\left[0\right.$ and $\quad v_{A^{I F S}}: X \rightarrow[0$, define intensity of integration function and the non-membership of element $\quad x \&$, respectively and $\quad 0 \leq \mu_{A^{I F S}}(x), v_{A^{I F S}}(x) \leq$, for every $\quad x \notin$.

Intuitionistic Fuzzy Numbers (IFN's): A subset of IFS, $A^{I F S}=\left\{\left\langle\left(x, \mu_{A^{I F S}}(x), v_{A^{I F S}}^{-}(x)\right\}: x \in\right.\right.$, of real line is called an IFN if following holds:

(i) $\exists m \in \Re_{s} \mu_{A^{I F S}}(m)=1$ and $v_{A^{I F S}}(m)=0$

(ii) $\mu_{A^{I F S}}^{-}: \Re \rightarrow[0,1]$ is continuous and for every $x \in \Re, 0 \leq \mu_{A^{I F S}}^{-}(x), v_{A^{I F S}}(x) \leq 1$ holds.

The membership function and non-membership function of Ais demonstrated,

$$
\mu_{A^{I F S}}(x)=\left\{\begin{array} { c } 
{ f _ { 1 } ( x ) , x \in [ m - \alpha _ { 1 } , } \\
{ 1 _ { s } \quad x = m } \\
{ h _ { 1 } ( x ) , x \in ( m , m + } \\
{ 0 , \quad \text { otherwise } }
\end{array} \quad v _ { A ^ { I F S } ( x ) } \quad \text { and } \left\{\begin{array}{c}
1_{s} \quad x \in\left(-\infty, m-\alpha_{2} .\right. \\
f_{2}(x), x \in\left[m-\alpha_{2}, m\right) \\
0, x=m, x \in\left[m+\beta_{2},\right. \\
h_{2}(x), x \in\left(m, m+\beta_{2}\right]
\end{array}\right.\right.
$$

Where $f_{\mathrm{i}}$ and $h_{\mathrm{i}}(x) ; i=$ are strictly increasing and decreasing functions in $\left[m-\alpha_{\mathrm{i}}\right.$, and $\left(m, m-\right.$ respectively. $\alpha_{i}$ and $\beta_{i}$ are the left and right spreads of $\mu_{A^{I F S}}^{-}$and $\quad v_{A^{I F S}}$ respectively.

Trapezoidal Intuitionistic Fuzzy Number (TrIFN): An IFN $\tilde{A}^{T r I F S}=\left\langle\left(a_{1}, a_{2}, a_{3}, a_{4}\right)\left(a_{1}^{\prime}, a_{2}, a_{3}, a_{4}^{\prime}\right)\right\rangle$ is a TrIFN in $\mathfrak{R}$ with the following membership function $\quad \mu_{i}$ and non-membership function $v_{2}$ defined by

$$
\mu_{\tilde{A}^{T F S}}(x)=\left\{\begin{array}{ll}
0 & x<a_{1} \\
\frac{x-a_{1}}{a_{2}-a_{1}} & a_{1}<x<a_{2} \\
1 & a_{2}<x<a_{3} \\
\frac{x-a_{4}}{a_{3}-a_{4}} & a_{3}<x<a_{4} \\
0 & a_{4}<x
\end{array} \quad \text { and } \quad v_{\tilde{A}^{I T S}}(x)= \begin{cases}0 & x<a_{1}^{\prime} \\
\frac{x-a_{2}}{a_{1}^{\prime}-a_{2}} & a_{1}^{\prime}<x<a_{2} \\
0 & a_{2}<x<a_{3} \\
\frac{x-a_{3}}{a_{3}-a_{4}^{\prime}} & a_{3}<x<a_{4}^{\prime} \\
1 & a_{4}^{\prime}<x\end{cases}\right.
$$

Generalized TrIFN (GTrIFN): An IFN $\tilde{A}^{I F S}=\left\langle\left(a_{1}, a_{2}, a_{3}, a_{4} ; \omega_{a}\right)\left(a_{1}^{\prime}, a_{2}, a_{3}, a_{4}^{\prime} ; \sigma_{a}\right)\right\rangle_{\text {claimed to be a }}$ GTrIFN if its integration and non-membership consequence are respectively liable

$$
\mu_{\tilde{A}^{T S}}(x)=\left\{\begin{array}{ll}
0 & \text { if } x<a_{1} \\
\frac{\left(x-a_{1}\right) \omega_{a}}{a_{2}-a_{1}} & \text { if } a_{1}<x<a_{2} \\
\omega_{a} & \text { if } a_{2}<x<a_{3} \\
\frac{\left(x-a_{4}\right) \omega_{a}}{a_{3}-a_{4}} & \text { if } a_{3}<x<a_{4} \\
0 & \text { if } a_{4}<x
\end{array} \quad \text { and } \quad v_{\tilde{A}^{T S}}(x)= \begin{cases}0 & \text { if } x<a_{1}^{\prime} \\
\frac{x-a_{2}-\sigma_{a}\left(a_{1}^{\prime}-x\right)}{a_{1}^{\prime}-a_{2}} & \text { if } a_{1}^{\prime}<x<a_{2} \\
\frac{\sigma_{a}}{a_{3}-x-\sigma_{a}\left(x-a_{4}^{\prime}\right)} & \text { if } a_{2}<x<a_{3} \\
a_{3}-a_{4}^{\prime} & \text { if } a_{3}<x<a_{4}^{\prime} \\
1 & \text { if } a_{4}^{\prime}<x\end{cases}\right.
$$

Where $\omega_{a}$ and $\sigma_{a}$ constitute extreme intensity of integration and minimal intensity of non-membership sequentially, gratifying $0 \leq \omega_{a} \leq 1,0 \leq \sigma_{a} \leq 1,0 \leq \omega_{a}+\sigma_{a} \leq 1$. Graphical representation of GTrIFN is illustrated in Fig.1. 


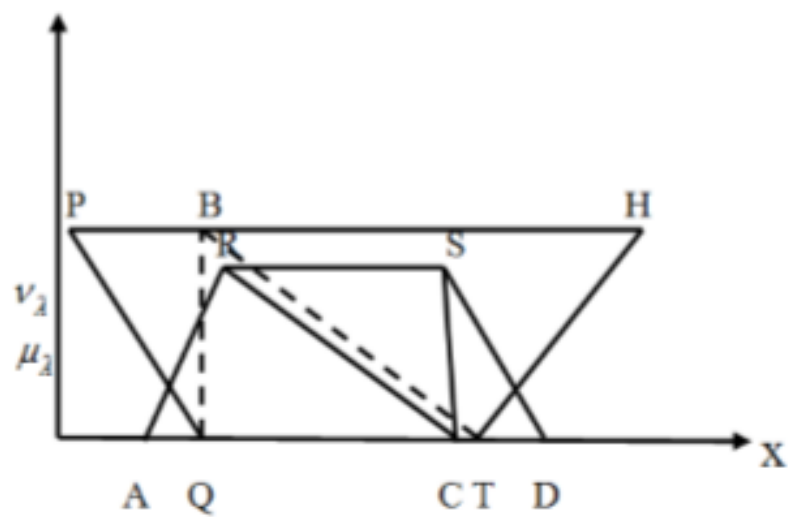

Fig 1: GTrIFN graph

\section{Arithmetic operations of GTrIFN:}

For any two TrIFN's

$\tilde{A}^{\text {TrIFN }}=\left\langle\left(a_{1}, a_{2}, a_{3}, a_{4} ; \omega_{a}\right)\left(a_{1}^{\prime}, a_{2}, a_{3}, a_{4}^{\prime} ; \sigma_{a}\right)\right\rangle$ and

$\tilde{B}^{\text {TrIFN }}=\left\langle\left(\mathrm{b}_{1}, \mathrm{~b}_{2}, \mathrm{~b}_{3}, \mathrm{~b}_{4} ; \omega_{b}\right)\left(\mathrm{b}_{1}^{\prime}, \mathrm{b}_{2}, \mathrm{~b}_{3}, \mathrm{~b}_{4}^{\prime} ; \sigma_{b}\right)\right\rangle$ the arithmetic operations are as follows,

(i) GTrIFN's Addition:

$\tilde{A}^{G T r I F N} \oplus \tilde{B}^{G T r I F N}=\left\langle\begin{array}{l}\left(a_{1}+b_{1}, a_{2}+b_{2}, a_{3}+b_{3}, a_{4}+b_{4} ; \min \left(\omega_{a}, \omega_{b}\right)\right) \\ \left(a^{\prime}{ }_{1}+b^{\prime}{ }_{1}, a_{2}+b_{2}, a_{3}+b_{3}, a^{\prime}{ }_{4}+b^{\prime}{ }_{4} ; \max \left(\sigma_{a}, \sigma_{b}\right)\right)\end{array}\right\rangle$

(ii) GTrIFN's Subtraction:

$\tilde{A}^{G T r I F N}-\tilde{B}^{G T r I F N}=\left\langle\begin{array}{l}\left(a_{1}-b_{4}, a_{2}-b_{2}, a_{3}-b_{3}, a_{4}-b_{1} ; \min \left(\omega_{a}, \omega_{b}\right)\right) \\ \left(a^{\prime}{ }_{1}-b^{\prime}{ }_{4}, a_{2}-b_{2}, a_{3}-b_{3}, a^{\prime}{ }_{4}-b_{1}^{\prime} ; \max \left(\sigma_{a}, \sigma_{b}\right)\right)\end{array}\right\rangle$

(iii) Scalar Multiplication:

$k \times \tilde{A}^{G T r I F N}=\left\langle\begin{array}{l}\left(k a_{1}, k a_{2}, k a_{3}, k a_{4} ; \omega_{a}\right)\left(k a_{1}^{\prime}, k a_{2}, k a_{3}, k a_{4}^{\prime} ; \sigma_{a}\right) \text { if } k>0 \\ \left(k a_{4}, k a_{3}, k a_{2}, k a_{1} ; \omega_{a}\right)\left(k a_{4}^{\prime}, k a_{3}, k a_{2}, k a_{1}^{\prime} ; \sigma_{a}\right) \text { if } k>0\end{array}\right\rangle$

\section{Ranking Function of GTrIFN}

Definition: Let the GTrIFN be $\tilde{A}^{\text {TrIFN }}=\left\langle\left(a_{1}, a_{2}, a_{3}, a_{4} ; \omega_{a}\right)\left(\mathrm{b}_{1}, \mathrm{~b}_{2}, \mathrm{~b}_{3}, \mathrm{~b}_{4} ; \omega_{a}\right)\right\rangle$ ranking function of a GTrIFN can be taken from Pardha Saradhi et al. [5] is

$$
R\left(\tilde{A}^{\text {TrIFN }}\right)=\left(\frac{a_{1}+b_{1}+2\left(a_{2}+b_{3}\right)+5\left(a_{3}+b_{2}\right)+a_{4}+b_{4}}{18}\right)\left(\frac{4 \omega_{a}+5 \omega_{b}}{18}\right)
$$

Ex: Let $\tilde{A}^{\text {GTrIFN }}=\langle(2,7,11,15 ; 0.5)(1,7,11,18 ; 0.3)\rangle$ then

$$
\begin{aligned}
R\left(\tilde{A}^{G T r I F N}\right) & =\left(\frac{2+1+2(7+11)+5(11+7)+15+18}{18}\right)\left(\frac{4(0.5)+5(0.3)}{18}\right) \\
& =1.75
\end{aligned}
$$

\section{Comparison of GTrIFN's:}

In order to compare GTrIFN's with every one obliged to grade. An assignment comparable $R: F(\Re)-$, which depict each TIFN's amongst existent rule, is called ranking function. At this moment, $F($ signify inclined GTrIFN's.

By using the ranking function " $R$ ", GTrIFN's can be compared.

Let

$$
\tilde{A}^{T r I F N}=\left\langle\left(a_{1}, a_{2}, a_{3}, a_{4} ; \omega_{a}\right)\left(a_{1}^{\prime}, a_{2}, a_{3}, a_{4}^{\prime} ; \sigma_{a}\right)\right\rangle
$$

and $\tilde{B}^{T r I F N}=\left\langle\left(\mathrm{b}_{1}, \mathrm{~b}_{2}, \mathrm{~b}_{3}, \mathrm{~b}_{4} ; \omega_{b}\right)\left(\mathrm{b}_{1}^{\prime}, \mathrm{b}_{2}, \mathrm{~b}_{3}, \mathrm{~b}_{4}^{\prime} ; \sigma_{b}\right)\right\rangle$ are two GTrIFN's then 


$$
\begin{array}{r}
R\left(\tilde{A}^{\text {TrIFN }}\right)=\left(\frac{a_{1}+a_{1}^{\prime}+2\left(a_{2}+a_{3}^{\prime}\right)+5\left(a_{3}+a_{2}^{\prime}\right)+a_{4}+a_{4}}{18}\right)\left(\frac{4 \omega_{a}+5 \sigma_{a}}{18}\right) \text { and } \\
R\left(\tilde{B}^{\text {TrIFN }}\right)=\left(\frac{b_{1}+b_{1}^{\prime}+2\left(b_{2}+b_{3}^{\prime}\right)+5\left(b_{3}+b_{2}^{\prime}\right)+b_{4}+b_{4}^{\prime}}{18}\right)\left(\frac{4 \omega_{b}+5 \sigma_{b}}{18}\right)
\end{array}
$$

Subsequently series circumscribed as

$$
\begin{aligned}
& \text { (i) } A^{\text {TYIFN }}>B^{\text {TYIFN }} \text { if } \mathrm{R}\left(A^{\text {TYIFN }}\right)>R\left(B^{\text {TYIFN }}\right) \text {. } \\
& \text { (ii) } A^{\text {TYIFN }}<B^{\text {TYIFN }} \text { if } \mathrm{R}\left(A^{\text {TYIFN }}\right)<R\left(B^{\text {TYWWN }}\right) \text {, and (iii) } A^{\text {TYIFN }}=B^{\text {TYIFN }} \text { if } \mathrm{R}\left(A^{\text {TYIFN }}\right)=\mathrm{R}\left(B^{\text {TYIFN }}\right)
\end{aligned}
$$

Ranking function $R$ confine the following possessions:

$$
\text { (i) } \mathrm{R}\left(A^{\text {TYIFN }}\right)+\mathrm{R}\left(B^{\text {TYIFN }}\right)=\mathrm{R}\left(A^{\text {TYIFN }}+B^{\text {TYL }} \text {, (ii) } \mathrm{R}\left(\mathrm{k} A^{\text {TYIFN }}\right)=\mathrm{k} \mathrm{R}\left(A^{\text {TYIFN }}\right) \forall \mathrm{k}\right. \text { \& }
$$

\section{Mathematical Formulation of Trapezoidal Intuitionistic Fuzzy Transportation problem:}

In TP decision maker or magnificent temporize abounding aspect over spanning in order through dealer and requirement. Occasionally decision maker is in decisive substantially more aggregate of peculiar commodity accessible at repository at peculiar time unlike intention. To this extent, he has not transmit to his associate or he is uncertain that how often aggregate of peculiar commodity credibly fabricate according to accessible primal matter by peculiar time. Uniformly, he may temporize from requirement. Intendedly new commodity eventually instigates in a market then he cannot decide exact aggregate of this commodity should transit to a peculiar terminus. Perhaps owing to unfamiliarity of the customers about this commodity or difference in cost and efficacy of commodity to similar one. We employ IFNs to deal with hesitation and uncertainty.

Appraise a TP with ' $m$ ' inceptions and ' $n$ ' terminus. Let $c_{i j}$ be cost of transiting one unit of commodity from inception to the terminus.

Let $\tilde{a}_{i}^{G T r I F N}=\left(a_{1}^{i}, a_{2}^{i}, a_{3}^{i}, a_{4}^{i}, \omega_{a} ; a_{1}^{i^{\prime}}, a_{2}^{i}, a_{3}^{i}, a_{4}^{i^{\prime}}, \sigma_{a}\right)$ be the IF quantity available at the origin.

$\tilde{b}_{j}^{G T r I F N}=\left(b_{1}^{j}, b_{2}^{j}, b_{3}^{j}, b_{4}^{j}, \omega_{b} ; b_{1}^{j^{\prime}}, b_{2}^{j}, b_{3}^{j}, b_{4}^{j^{\prime}}, \sigma_{b}\right)$ be the IF quantity needed at the destination. $\tilde{x}_{i j}^{G T r I F N}=\left(x_{1}^{i j}, x_{2}^{i j}, x_{3}^{i j}, x_{4}^{i j} ; x_{1}^{i j^{\prime}}, x_{2}^{i j}, x_{3}^{i j}, x_{4}^{i j^{\prime}}\right)$ be the IF quantity transformed from the origin to the destination. Then the balanced generalized trapezoidal intuitionistic fuzzy transportation problem is given by

$$
\begin{aligned}
\operatorname{Min} \tilde{Z}^{G T r I F N}= & \sum_{i=1}^{m} \sum_{j=1}^{n} c_{i j} \times x_{i j} \\
\text { s.t. } & \sum_{j=1}^{n} \tilde{x}_{i j}^{G T r I F N} \leq \tilde{a}_{i}^{I}, i=1,2, \ldots, m \\
& \sum_{i=1}^{m} \tilde{x}_{i j}^{G T r I F N} \geq \tilde{b}_{i}^{I}, j=1,2, \ldots, n \\
& \tilde{x}_{i j}^{G T r I F N} \geq \tilde{0} ; i=1,2, \ldots, m ; j=1,2, \ldots, n
\end{aligned}
$$

\section{Proposed Transportation strategy}

Preferred method is elementary and prompt strategy to seek prime solution $\left\{x_{i j}\right\}$ including intuitionistic fuzzy optimal value $\tilde{Z}^{G T r I F N}$ of TP having repository including demand limitations as real number and TC, $\tilde{c}_{i j}^{G T r I F N} ;(i=1,2, \ldots, m ; j=1,2, \ldots, n)$ from $i^{\text {th }}$ source to $j^{\text {th }}$ requirement, extract IFN represented in Table 1. 
Table 1 GrIFTP

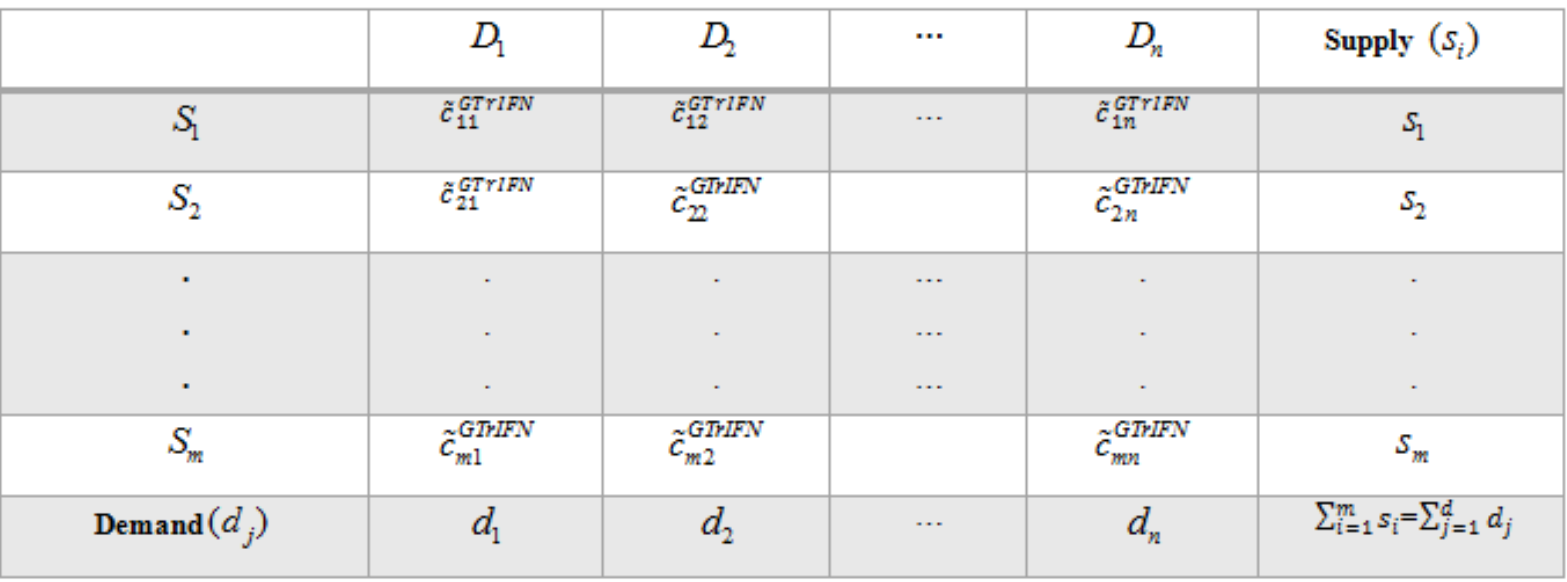

Stage 1: Utilizing separation formula, considered in "Comparison of IFTN's" segment, adopt least and greatest IFN from each archive and segment of intuitionistic fuzzy price matrix of TIFTP of type-2 and deduct it from each IFN's of their relating line and segment.

Stage 2: Find sum of row difference and column difference and denote row sum by R and column sum by C. Identify Maximum sum of row and column. Select maximum difference in row and column.

Stage 3: Choose the cell having most minimal expense in row and column identified in stage 2.

Stage 4: Make a feasible assignment to the cell picked in stage 5. Delete fulfilled row/column.

Stage 5: Repeat the technique until all the designations has been made.

Stage 6: The Optimum solution and triangular intuitionistic optimum value is attained in stage 8 , is optimum solution \{\} and t triangular intuitionistic fuzzy optimum value is $\sum_{i=1}^{m} \sum_{j=1}^{n} c_{i j} \mathbb{Q}$

\section{Numerical Examples:}

In this unit, a prevailed numerical example is resolved to instance proposed generalized trapezoidal intuitionistic fuzzy zero centred method.

Example 1: An existing Generalized trapezoidal Intuitionistic fuzzy transportation problem (GTrIFTP), with three repositories i.e. $S_{1}, S_{2}, S_{a}$ including three demands i.e., $D_{1}, D_{2}, D_{a}$ respectively by Table 2 taken from Shashi et al. [7], is resolved using proposed Transportation strategy.

Table 2 GTrIFTP

\begin{tabular}{|c|c|c|c|c|}
\hline & $D_{1}$ & $D_{2}$ & $D_{3}$ & Supply \\
\hline$S_{1}$ & $(2,4,8,15 ; 0.6)(1,4,8,18: 0.3)$ & $(3,5,7,12 ; 0.5)(1,5,7,15 ; 0.3)$ & $(2,5,9,16 ; 0.7)(1,5,9,18 ; 0.3)$ & 25 \\
\hline$S_{2}$ & $(2,5,8,10 ; 0.6)(1,5,8,12 ; 0.2)$ & $(4,8,10,13 ; 0.4)(3,8,10,15 ; 0.3)$ & $(3,6,10,15 ; 0.8)(2,6,10,18 ; 0.2)$ & 30 \\
\hline$S_{3}$ & $(2,7,11,15 ; 0.5)(1,7,11,18 ; 0.3)$ & $(5,9,12,16 ; 0.7)(3,9,12,19 ; 0.2)$ & $(4,6,8,10 ; 0.6)(3,6,8,12 ; 0.3)$ & 40 \\
\hline Demand & 35 & 45 & 15 & \\
\hline
\end{tabular}

Solution: Problem is resolved in the following stages

Select maximum and minimum TIFN in each row and column take the difference as given in table 3. 
Table 3 Row and Column Difference Table

\begin{tabular}{|c|c|c|c|c|c|}
\hline & $D_{1}$ & $D_{2}$ & $D_{3}$ & Supply & $\begin{array}{c}\text { Row } \\
\text { difference }\end{array}$ \\
\hline$S_{1}$ & $(2,4,8,15 ; 0.6)$ & $(3,5,7,12 ; 0.5)$ & $(2,5,9,16 ; 0.7)$ & 25 & 0.22 \\
& $(1,4,8,18: 0.3)$ & $(1,5,7,15 ; 0.3)$ & $(1,5,9,18 ; 0.3)$ & & 0.36 \\
\hline$S_{2}$ & $(2,5,8,10 ; 0.6)$ & $(4,8,10,13 ; 0.4)$ & $(3,6,10,15 ; 0.8)$ & 30 & \\
& $(1,5,8,12 ; 0.2)$ & $(3,8,10,15 ; 0.3)$ & $(2,6,10,18 ; 0.2)$ & & 0.76 \\
\hline$S_{3}$ & $(2,7,11,15 ; 0.5)$ & $(5,9,12,16 ; 0.7)$ & $(4,6,8,10 ; 0.6)$ & 40 & \\
& $(1,7,11,18 ; 0.3)$ & $(3,9,12,19 ; 0.2)$ & $(3,6,8,12 ; 0.3)$ & & 1.34 \\
\hline Demand & 35 & 45 & 15 & 95 & 1.59 \\
Column & 0.50 & 0.81 & 0.28 & & \\
\hline
\end{tabular}

The problem given in Table 3, transformed in Table 4 by using the Stage 2 and assign first allocation using stage 4 of proposed method.

Table 4 First allocation Table

\begin{tabular}{|c|c|c|c|c|c|c|}
\hline & $D_{1}$ & $D_{2}$ & & $D_{3}$ & Supply & $\begin{array}{c}\text { Row } \\
\text { difference }\end{array}$ \\
\hline$S_{1}$ & $\begin{array}{l}(2,4,8,15 ; 0.6) \\
(1,4,8,18: 0.3)\end{array}$ & $\begin{array}{l}(3,5,7,12 ; 0.5) \\
(1,5,7,15 ; 0.3)\end{array}$ & 25 & $\begin{array}{l}(2,5,9,16 ; 0.7) \\
(1,5,9,18 ; 0.3)\end{array}$ & $\begin{array}{c}25 \\
0\end{array}$ & 0.22 \\
\hline$S_{2}$ & $\begin{array}{l}(2,5,8,10 ; 0.6) \\
(1,5,8,12 ; 0.2)\end{array}$ & $\begin{array}{l}(4,8,10,13 ; 0.4) \\
(3,8,10,15 ; 0.3)\end{array}$ & & $\begin{array}{l}(3,6,10,15 ; 0.8) \\
(2,6,10,18 ; 0.2)\end{array}$ & 30 & 0.36 \\
\hline$S_{3}$ & $\begin{array}{l}(2,7,11,15 ; 0.5) \\
(1,7,11,18 ; 0.3)\end{array}$ & $\begin{array}{l}(5,9,12,16 ; 0.7) \\
(3,9,12,19 ; 0.2)\end{array}$ & & $\begin{array}{l}(4,6,8,10 ; 0.6) \\
(3,6,8,12 ; 0.3)\end{array}$ & 40 & 0.76 \\
\hline Demand & 35 & $\begin{array}{l}45- \\
20\end{array}$ & & 15 & 95 & 1.34 \\
\hline $\begin{array}{c}\text { Column } \\
\text { difference }\end{array}$ & 0.50 & 0.81 & & 0.28 & 1.59 & \\
\hline
\end{tabular}

Using stage 4 of proposed method remove from Table 4. New reduced shown in Table 5 again apply the procedure

Table 5 New Reduced Table

\begin{tabular}{|c|c|c|c|c|c|}
\hline & $D_{1}$ & $D_{2}$ & $D_{3}$ & $\begin{array}{c}\text { Supply } \\
\text { difference }\end{array}$ \\
\hline$S_{2}$ & $(2,5,8,10 ; 0.6)$ & $(4,8,10,13 ; 0.4)$ & $(3,6,10,15 ; 0.8)$ & 30 & 0.36 \\
& $(1,5,8,12 ; 0.2)$ & $(3,8,10,15 ; 0.3)$ & $(2,6,10,18 ; 0.2)$ & & 0.76 \\
\hline$S_{3}$ & $(2,7,11,15 ; 0.5)$ & $(5,9,12,16 ; 0.7)$ & $(4,6,8,10 ; 0.6)$ & 40 & \\
& $(1,7,11,18 ; 0.3)$ & $(3,9,12,19 ; 0.2)$ & $(3,6,8,12 ; 0.3)$ & & 1.12 \\
\hline Demand & 35 & 20 & 0.28 & 1.06 & \\
\hline Column & 0.50 & 0.28 & & & \\
\hline
\end{tabular}


Table 6 Second Allocation table

\begin{tabular}{|c|c|c|c|c|c|}
\hline & D1 & D2 & D3 & Supply & $\begin{array}{c}\text { Row } \\
\text { difference }\end{array}$ \\
\hline$S_{2}$ & $\begin{array}{l}(2,5,8,10 ; 0.6) \\
(1,5,8,12 ; 0.2)\end{array}$ & $\begin{array}{l}(4,8,10,13 ; 0.4) \\
(3,8,10,15 ; 0.3)\end{array}$ & $\begin{array}{l}(3,6,10,15 ; 0.8) \\
(2,6,10,18 ; 0.2)\end{array}$ & 30 & 0.36 \\
\hline$S_{3}$ & $\begin{array}{l}(2,7,11,15 ; 0.5) \\
(1,7,11,18 ; 0.3)\end{array}$ & $\begin{array}{l}(5,9,12,16 ; 0.7) \\
(3,9,12,19 ; 0.2)\end{array}$ & $\begin{array}{l}(4,6,8,10 ; 0.6) \\
(3,6,8,12 ; 0.3)\end{array}$ & 25 & 076 \\
\hline Demand & 35 & 20 & $\begin{array}{c}15 \\
0\end{array}$ & 95 & 1.12 \\
\hline $\begin{array}{c}\text { Column } \\
\text { difference }\end{array}$ & 0.50 & 0.28 & 0.28 & 1.06 & \\
\hline
\end{tabular}

Again applying the Stage 5 of the proposed method, all the allocations are made as shown in Table 7.

Table 7 Final allocation table

\begin{tabular}{|c|c|c|c|}
\hline & $D_{1}$ & $D_{2}$ & $D_{3}$ \\
\hline$S_{1}$ & $(2,4,8,15 ; 0.6)(1,4,8,18: 0.3)$ & $(3,5,7,12 ; 0.5)(1,5,7,15 ; 0.3)$ & $(2,5,9,16 ; 0.7)(1,5,9,18 ; 0.3)$ \\
& & $\mathbf{2 5}$ & \\
\hline$S_{2}$ & $(2,5,8,10 ; 0.6)(1,5,8,12 ; 0.2)$ & $(4,8,10,13 ; 0.4)(3,8,10,15 ; 0.3)$ & $(3,6,10,15 ; 0.8)(2,6,10,18 ; 0.2)$ \\
& $\mathbf{3 0}$ & $(5,9,12,16 ; 0.7)(3,9,12,19 ; 0.2)$ & $(4,6,8,10 ; 0.6)(3,6,8,12 ; 0.3)$ \\
\hline$S_{3}$ & $(2,7,11,15 ; 0.5)(1,7,11,18 ; 0.3)$ & $\mathbf{2 0}$ & $\mathbf{1 5}$ \\
\hline
\end{tabular}

\section{Stage 6: Optimum solution and IF optimum value}

Optimal solution, attained in Stage 4 , is $x_{12}=25, x_{21}=30, x_{31}=5, x_{32}=20$ and $x_{33}=15$. Generalized trapezoidal Intuitionistic fuzzy optimum value of trapezoidal Intuitionistic fuzzy transportation problem, given in Table 2, is

$$
\begin{aligned}
& 25 \otimes(3,5,7,12 ; 0.5)(1,5,7,15 ; 0.3)+30 \otimes(2,5,8,10 ; 0.6)(1,5,8,12 ; 0.2)+5 \otimes(2,7,11,15 ; 0.5)(1,7,11,18 ; 0.3) \\
& +20 \otimes(5,9,12,16 ; 0.7)(3,9,12,19 ; 0.2)+15 \otimes(4,6,8,10 ; 0.6)(3,6,8,12 ; 0.3) \\
& =(305,580,830,1145 ; 0.5)(165,580,830,1385 ; 0.3) \approx 139.0278 .
\end{aligned}
$$

\section{Conclusions}

Finally we initiate an optimum solution for generalized trapezoidal intuitionistic fuzzy transportation problem whose costs are taken as GTrIFN's. In initiated method we solved by using ranking function found by PardhaSaradhi et al. [5]. Using this method we can attain directly optimum solution without finding IBFS, which is simplest method and we can solve real life transportation problems.

\section{References}

Atanassov, K.T., "Intuitionistic fuzzy sets”, Fuzzy Sets Syst. 20, 87-96(1986).

Bellman,R.,Zadeh,L.A., "Decision making in fuzzy environment”, Manag. Sci.17(B),141-164,(1970).

Chakraborty,D.,Jana,D.K.,Roy,T.K., "Arithmetic operations on generalized intuitionistic fuzzy number and its applications to transportation problem”, Opsearch52, 431-471,(2015).

Ismail Mohideen.S, Senthil Kumar. A, "A Comparative Study on Transportation Problem in fuzzy environment”, International Journal of Mathematics Research, Vol. 2 Number.1,pp. 151-158,(2010).

Pardhasaradhi. B, Madhuri. M. V. and Ravi Shankar. N, "Ordering of Intuitionistic fuzzy numbers using centroid of centroids of Intuitionistic fuzzy numbers", International Journal of Mathematics Trends and Technology, Vol. 52, No. 5, pp.276-285, (2017).

Stephen Dinager. D, Palanivel. K, "The Transportation problem in fuzzy environment”, International Journal of Algorithm, computing and mathematics, Vol. 2, No. 3, (2009). 
Shashi Aggarwal, Chavi Gupta, "Algorithm for Solving Intuitionistic Fuzzy Transportation Problem with Generalized Trapezoidal Intuitionistic Fuzzy Number via New Ranking Method, Algorithm For Intuitionistic Fuzzy Transportation Problem", (2015).

Wang, J., Zhang, Z., "Aggregation operators on Intuitionistic trapezoidal Fuzzy Numbers and its applications to Multi-Criteria Decision Making Problems", J. Syst. Eng. Elect. 20, 321-326, (2009).

Wang, X., Kerre, E.E., "Reasonable properties for the ordering of fuzzy quantities (I)", Fuzzy Sets Syst. 118, 375-385, (2001).

Wang J Q., "Survey on fuzzy multi-criteria decision making approach”, Control and Decision, 2008(2).

Zadeh, L.A., "Fuzzy sets”, Inf. Comput. 8, 338-353, (1965). 